\title{
Fisher's Zeros and Perturbative Series in Gluodynamics
}

\author{
A. Denbleyker, D. Du, and Y. Meurice* \\ Department of Physics and Astronomy, The University of Iowa, Iowa City, Iowa 52242, USA \\ E-mail: alan-denbleyker@uiowa.edu \\ E-mail: daping-du@uiowa.edu \\ E-mail:yannick-meurice@uiowa.edu
}

\section{A. Velytsky}

Department of Physics and Astronomy, UCLA, Los Angeles, CA 90095-1547, USA

E-mail: velephysics.ucla.edu

\begin{abstract}
We study the zeros of the partition function in the complex $\beta$ plane (Fisher's zeros) in SU(2) and SU(3) gluodynamics. We discuss their effects on the asymptotic behavior of the perturbative series for the average plaquette. We present new methods to infer the existence of these zeros in region of the complex $\beta$ plane where $\mathrm{MC}$ reweighting is not reliable. These methods are based on the assumption that the plaquette distribution can be approximated by a $\phi^{4}$ type distribution. We give new estimates of the locations for a $4^{4}$ lattice. For $S U(2)$, we found zeros at $\beta=2.18(1) \pm$ $i 0.18$ (2) (which differs from previous estimates), and at $\beta=2.18(1) \pm i 0.22(2)$. For $S U(3)$, we confirm $\beta=5.54(2) \pm i 0.10(2)$ and found additional zeros at $\beta=5.54(2) \pm i 0.16(2)$. Some of the technical material can be found in recent preprints, in the following we emphasize the motivations (why it is important to know the locations of the zeros) and the challenges (why it is difficult to locate the zeros when the volume increases).
\end{abstract}

The XXV International Symposium on Lattice Field Theory

July 30-4 August 2007

Regensburg, Germany

\footnotetext{
* Speaker.

${ }^{\dagger}$ Current address: EFI, University of Chicago, 5640 S. Ellis Ave., Chicago, IL 60637 and Argonne National Laboratory, 9700 Cass Ave., Argonne, IL 60439
} 


\section{Introduction}

More than 200 years ago, L. Euler [1] realized that the asymptotic behavior of the expansions coefficients of the Bessel functions about the origin could be obtained from the location of the complex zeros. For a single plaquette with $S U(2)$ gauge group (as defined, for instance, in Ref. [2], as usual $\beta=4 / g^{2}$ ), we obtain that in this simple model the average plaquette $P$ can be written as

$$
P=1-2 \beta \sum_{n=1}^{\infty} \frac{1}{\beta^{2}+\alpha_{n}^{2}}
$$

where the $\alpha_{n}$ are the locations of the zeros of $J_{1}(z)$ (on the positive real axis). Using the geometrical series one sees that the radius of convergence of the expansion in $\beta$ is $1 / \alpha_{1}$. This result can be rephrased in the following way: the radius of convergence of the strong coupling expansion of $P$ is the distance from the origin to the closest zero of the partition function (which in this simple example is located, as well as all the other zeros, on the imaginary axis).

The situation is more interesting at large $\beta$ and fundamentally different from the case of scalar models. As well known, the zeros of $J_{1}(z)$ become approximately equally spaced for large real argument. If we now consider $P$ in the $g^{2}=4 / \beta$ complex plane, the zeros accumulate at zero along the imaginary axis which plays the role of a Stokes line. Unlike the scalar case the partition function is well defined at real negative $g^{2}$, but as we cross the imaginary axis, the values of $P$ changes discontinuously. This can be seen from the sum rule $P(\beta)+P(-\beta)=2$, which remains true on arbitrary lattices with even number of sites in every directions [3].

We expect that the features of the strong and weak coupling expansions observed for the one plaquette model to remain true on generic lattices. However, other zeros of the partition function related to phase transitions are expected. For the 2-dimensional Ising model, it has been observed by M. Fisher [4] that the zeros of the partition function pinch the real axis of the complex inverse temperature at $\beta_{c}$. For the 3-dimensional Ising model, numerical calculations consistent with this scenario have been obtained [5] on a $4^{3}$ lattice. These zeros in the complex $\beta$ plane are called Fisher's zeros and should be distinguished from the Lee-Yang zeros in the complex magnetic field plane.

For a zero temperature lattice gauge theory with a Wilson action, we expect no phase transition on the real $\beta$ axis. However, such theories can be seen as "close" to other theories (e. g. at non-zero temperature or with a positive adjoint coupling) that have a phase transition. Consequently, it is plausible that zero temperature lattice gauge theory with a Wilson action have Fisher zeros close to the real axis but that these zeros do not pinch the real axis in the infinite volume limit. This is a plausible explanation [6] for the unexpected behavior [7] of the weak coupling expansion of $P$ for $S U(3)[8,9]$. Standard methods of series analysis suggest $[7,6]$ a singularity on the real axis, namely $P \propto(1 / 5.74-1 / \beta)^{1.08}$. This would imply a peak in the second derivative of $P$ with a height increasing with the volume, which is not seen at zero temperature [6]. The vicinity of the critical point in the fundamental-adjoint plane, suggests the approximate mean field behavior [6]:

$$
-\partial P / \partial \beta \propto \ln \left(\left(1 / \beta_{m}-1 / \beta\right)^{2}+\Gamma^{2}\right),
$$

Fits of the series with such parametric form yield the approximate values $\beta_{m} \simeq 5.78$ and $\Gamma \simeq 0.006$ (i.e $\operatorname{Im} \beta \simeq 0.2$ ). 
Values of $\Gamma$ which are too large (too small) would produce modulations of the coefficients (peaks in the derivatives of $P$ ) which are not observed. A detailed analysis [6] yield the bounds (for $S U(3)) 0.001<\Gamma<0.01$. This suggests zeroes of the partition function in the complex $\beta$ plane with

$$
0.03 \simeq 0.001 \beta_{m}^{2}<\operatorname{Im} \beta<0.01 \beta_{m}^{2} \simeq 0.33
$$

In these proceedings, we report recent efforts to locate such zeros in pure gauge theories with gauge group $S U(2)$ and $S U(3)$. Some of the technical details can be found in a recent preprint [11]. Another preprint is in preparation [12] and should be available soon. In order to avoid repetitions, we will emphasize the motivations and aspects not covered in these preprints.

\section{The non-perturbative part of the plaquette and the gluon condensate}

It is quite common that the difference between a physical quantity and its perturbative expansion is of the form $\exp \left(-K / g^{2}\right)$. One of the best known example is the quantum mechanical double-well where the perturbative series is not able to take into account the tunneling effect and instantons are needed. For the average plaquette, the issue is obscured by the hypothetical zero close to the real axis and the factorial growth necessary to get an envelope [10] in the accuracy versus coupling at successive order is not reached at the order where the perturbative expansion is available. Larger order extrapolation are necessary. Two models have been considered [10].

The first is based on Eq. (1.2) which implies a dilogarithmic series for $P$ :

$$
P \sim \sum_{k=0} a_{k} \beta^{-k} \simeq C\left(\operatorname{Li}_{2}\left(\beta^{-1} /\left(\beta_{m}^{-1}+i \Gamma\right)\right)+\text { h.c } .\right.
$$

The low order coefficients depend very little on $\Gamma$ as long as the upper bound given above is satisfied. It is remarkable that the method of stochastic perturbation theory allows us to calculate the series up to order 10 [8] and 16 [9], however larger series are needed to resolve $\Gamma$ ! As we will explain below, the situation might be better for $S U(2)$. The two other parameters can be determined using for instance the values of $a_{9}$ and $a_{10}$. This gives very good predictions [10] of the values of $a_{8}, a_{7}, \ldots$. This nice regularity is still begging for a diagrammatic explanation.

Despite its predictive success, the dilogarithmic series has a finite radius of convergence and the coefficients do not have the expected factorial growth that is observed in the one plaquette model. The second extrapolation was based on an IR renormalon model [13, 14, 15]

$$
\begin{gathered}
\sum_{k=0} a_{k} \bar{\beta}^{-k} \simeq K \int_{0}^{\infty} d t \mathrm{e}^{-\bar{\beta} t}\left(1-t 33 / 16 \pi^{2}\right)^{-1-204 / 121} \\
\bar{\beta}=\beta\left(1+d_{1} / \beta+\ldots\right)
\end{gathered}
$$

These two extrapolations seem consistent with the behavior

$$
P(\beta)-P_{\text {pert. }}(\beta) \simeq C\left(a / r_{0}\right)^{4}
$$

with $a(\beta)$ defined with the force scale $[16,17]$ with $r_{0}=0.5 \mathrm{fm}$, and $P_{\text {pert }}$ appropriately truncated. For large $\beta$ this has the desired exponential form. Attempts have been made in the past $[18,7,9]$ to relate $C$ to the so-called gluon condensate [14]. Remembering the $\alpha / \pi$ factor in the definition, 


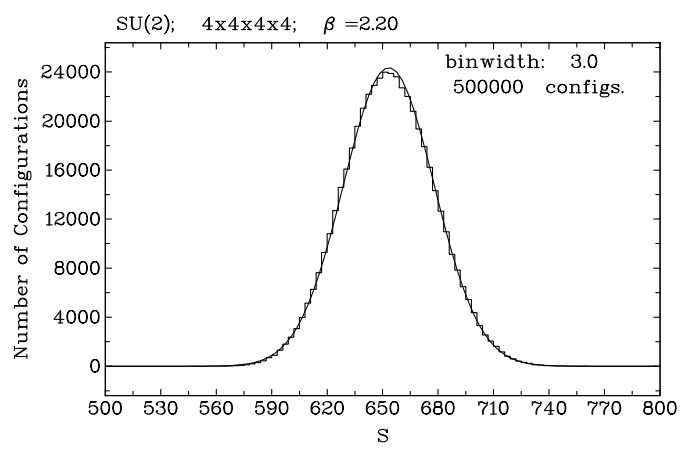

Figure 1: Distribution of the 500,000 values of $S$ in an histogram with 100 bins for a $S U(2)$ pure gauge theory on a $4^{4}$ lattice at $\beta=2.20$. The solid line is a Gaussian fit.

the value that could in principle be compared with the commonly used value of $0.012 \mathrm{GeV}^{4}$ is $\left(36 / \pi^{2}\right) C r_{0}^{-4}$ for $N_{c}=3 . C$ is sensitive to resummation. $C \simeq 0.6$ with the bare series [10] and 0.4 with the tadpole improved series [9]. This gives values 3-5 times larger than the value quoted above. Besides the question of scheme dependence, the gluon condensate is not an order parameter and it seems difficult to compare the lattice results with quantities defined in the context of sum rules. On the other hand, it is important to figure out how well the scaling with the lattice spacing given in Eq. (2.4) is obeyed and if it can be explained semi-classically as it can be done for the double-well. In this context, it is crucial to understand the complex singularities of $P$, which complicate the analysis of the scaling.

\section{The zeros of the partition function}

The zeros of the partition function can be located with the reweighting method [19, 20].

$$
Z\left(\beta_{0}+\Delta \beta\right)=Z\left(\beta_{0}\right)<\exp (-\Delta \beta S)>_{\beta_{0}} .
$$

It is convenient to subtract $\langle S\rangle$ from $S$ in the exponential because it removes fast oscillations without changing the complex zeroes. $Z(\beta)$ is the Laplace transform of density of states $n(S)$ :

$$
Z(\beta)=\int_{0}^{\infty} d S n(S) \exp (-\beta S)
$$

If we could estimate $Z(\beta)$ along an axis in the imaginary direction, we could calculate the inverse Laplace transform and obtain $n(S)$. As shown in Fig. 1, the distribution of values of $S$ for $S U(2)$ can be fitted very well with a Gaussian with $\left.\sigma_{S}^{2}=<S^{2}>-<S\right\rangle^{2}$. This suggests [20] a criterion to determine a region of confidence for MC zeros. For a Gaussian distribution, the fluctuation in $\exp (-\Delta \beta(S-<S>))$ is smaller than the average for $|\Delta \beta|^{2}<\ln \left(N_{\text {conf. }}\right) / \sigma_{S}^{2}$. This defines a radius of confidence $\sqrt{\ln \left(N_{\text {conf. }}\right)} / \sigma_{S}$ in the complex $\beta$ plane. As $\sigma_{S}^{2} \propto V$, the radius of confidence shrinks like $V^{-1 / 2}$ which is hard to beat with $\sqrt{\ln N_{\text {conf. }} \text {. }}$.

When the zeros pinch the real axis as the volume increases, which is expected near a phase transition, there is some hope that it is possible to follow them with MC methods. This explains 

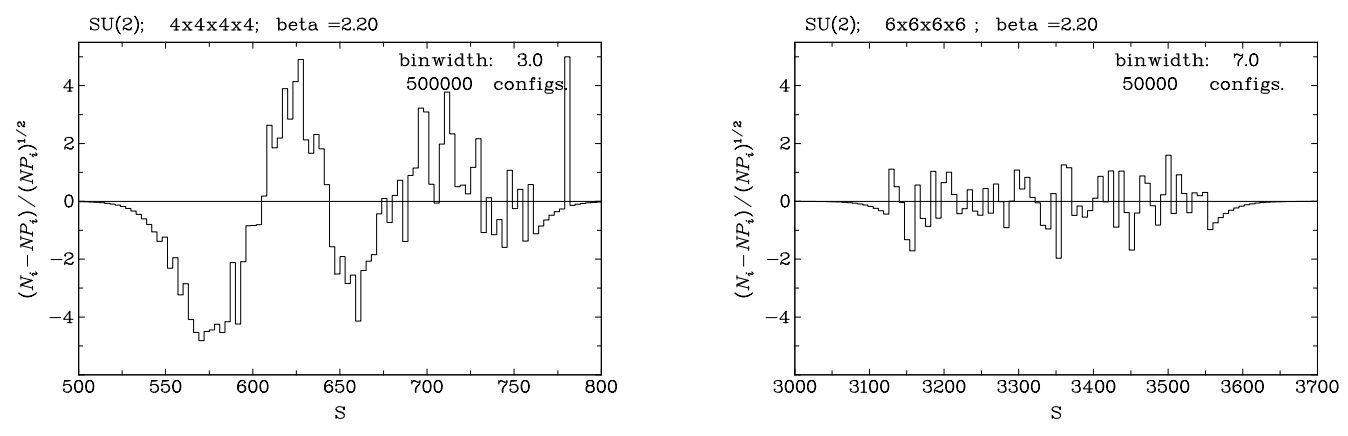

Figure 2: $r_{i}=\left(N_{i}-N P_{i}\right) / \sqrt{N P_{i}}$ for $S U(2)$ at $\beta=2.20$ on $4^{4}$ and $6^{4}$ lattices.

some positive results [20] on $2 \times L^{3}$ and $4 \times L^{3}$ lattices. On the other hand, on $L^{4}$ lattices, we expect the zeros to stabilize away from the real axis and MC methods can only give lower bounds that shrink when $L$ increases. For instance for $S U(3)$ on a $8^{4}$ lattice [21], we were only able to verify that $\operatorname{Im} \beta>0.03$. In addition, for $S U(2)$, there seem to be no zeros in the Gaussian region of confidence even on a $4^{4}$ lattice. For these reasons we have developed new methods to find zeros of the partition that lay outside of the region of confidence of MC calculations.

\section{New methods to locate the zeros $[11,12]$}

Gaussian distributions (of $S$ ) have no complex zeros. The Gaussian circle of confidence in the complex $\beta$ plane defined by the condition $\sigma_{f}<|f| \sqrt{N_{\text {conf }} \text {. }}$. If this criterion is applied directly to a non-Gaussian distribution having complex zeros, it will automatically exclude the regions that contain the zeros. When looking for complex zeros, we look for the intersection of the zero level curves for the real and imaginary parts. We are interested in knowing how much these level curves can move due to statistical fluctuations. We proposed [11] to consider the alternative region of confidence defined by a condition that controls the error on the level curves:

$$
\sigma_{f}<d \sqrt{N_{\text {conf }}}\left|f^{\prime}\right|
$$

In order to be useful $d$ should be a fraction of the typical distance between zero level curves of the real and imaginary part. This methods has allowed [11] to reject dubious zeros on the edge of the Gaussian circle of confidence for $S U(2)$ on a $4^{4}$ lattice. It remains applicable when the deviation from a Gaussian distribution is significant and true zeros appear.

The zeros come from the deviations from the Gaussian behavior. As shown on Fig. 2, discrepancies in unit of the expected fluctuations are coherent for $L=4$ but as the the volume increases, the signal gets lost in the noise (for that particular value of $\beta$ ). The nice regularities of the difference with the Gaussian approximation (for small lattices) suggest to fit the distribution with

$$
P(S) \propto \exp \left(-\lambda_{1} S-\lambda_{2} S^{2}-\lambda_{3} S^{3}-\lambda_{4} S^{4}\right)
$$

The unknown parameters were determined from the first four moments using Newton's methods and also by $\chi^{2}$ minimization. Very good agreement between the two methods was found on $4^{4}$ 

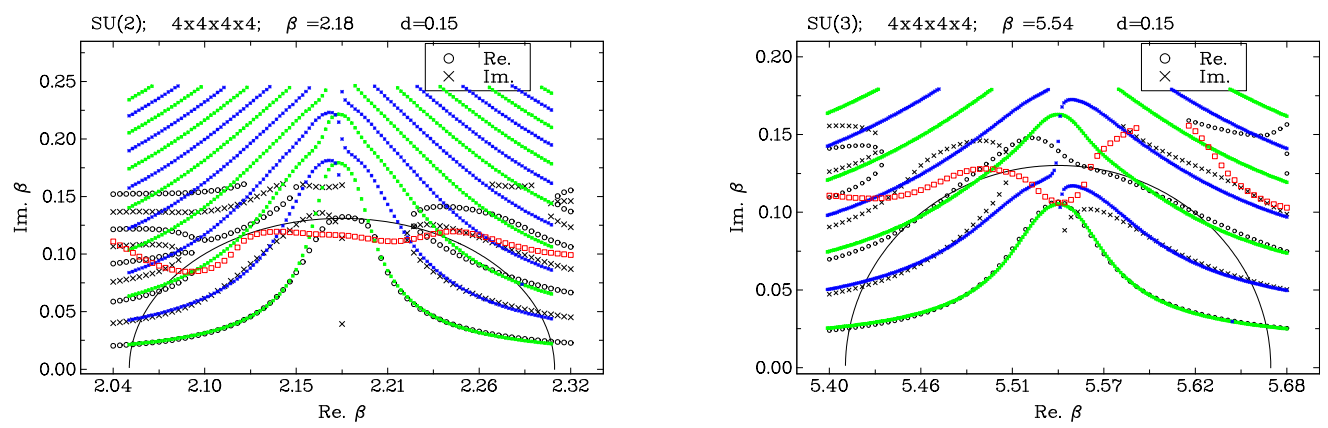

Figure 3: Zeros of the real (crosses) and imaginary (circles) using MC on a $4^{4}$ lattice, for $S U(2)$ at $\beta=$ 2.18 and $S U(3)$ at $\beta=5.54$. The smaller dots are the values for the real (green) and imaginary (blue) parts obtained from the 4 parameter model. The MC exclusion region boundary for $d=0.15$ is represented by boxes (red).

lattices. The zeros can then be calculated for the parametric form (4.2) using accurate numerical integration. The results are shown in Fig. 3 on a $4^{4}$ lattice. Comparing results at different $\beta$ on a $4^{4}$ lattice, we obtained the following locations of the complex zeros:

- $\beta=2.18(1) \pm i 0.18$ (2) for $S U$ (2) (differs from [19] 2.23 $\pm i 0.155$ obtained with MC outside regions of confidence) and another zero at $\beta=2.18(1) \pm i 0.22(2)$.

- $\beta=5.54(2) \pm i 0.10(2)$ for $S U(3)$ (agrees with [20]) and another zero at $\beta=5.54(2) \pm$ $i 0.16(2)$.

Note that the ratio of the imaginary and real parts of the closest zero is almost 5 times larger in $S U(2)$. This indicates that modulations in the perturbative coefficients of $P$ should be easier to see than in $S U(3)$.

\section{Conclusions}

We have build a ladder of methods that can be applied for increasing values of the imaginary part. We found new ways to distinguish fake and true MC zeros that work well with non-Gaussian examples. Fitting methods based on cubic and quartic perturbations give consistent results at different $\beta$ for larger values of the imaginary part on a $4^{4}$ lattice. Results on larger lattices will be available soon [12]. We are in the process of checking the selfconsistency of the parametrization at different $\beta$ and are attempting to extract the density of states. Effect of an adjoint term, finite-temperature and decimation are also under study.

\section{Acknowledgments}

This research was supported in part by the Department of Energy under Contract No. FG0291ER40664 and the National Science Foundation NSF-PHY-0555693. 


\section{References}

[1] L. Euler, Acta Acad. Petrop. 1781, p. 170.

[2] L. Li and Y. Meurice, An example of optimal field cut in lattice gauge perturbation theory, Phys. Rev. D71 (2005) 054509, [hep-lat/ 0501023$].$

[3] L. Li and Y. Meurice, Lattice gluodynamics at negative g², Phys. Rev. D71 (2005) 016008, [hep-lat/0410029].

[4] M. Fisher, Lectures in Theoretical Physics Vol. VIIC, University of Colorado Press (1965).

[5] R. Pearson, Partition function of the Ising model on the periodic 4x4x4 lattice, Phys. Rev., B26 (1982) 6285.

[6] L. Li and Y. Meurice, About a possible 3rd order phase transition at T = 0 in 4D gluodynamics, Phys. Rev. D73, 036006 (2006), [hep-lat/ 0507034 ].

[7] R. Horsley, P. E. L. Rakow, and G. Schierholz, Separating perturbative and non-perturbative contributions to the plaquette, Nucl. Phys. Proc. Suppl. 106 (2002) 870-872, [hep-lat/0110210].

[8] F. Di Renzo and L. Scorzato, A consistency check for renormalons in lattice gauge theory: $\beta^{-10}$ contributions to the su(3) plaquette, JHEP 10 (2001) 038, [hep-lat/0011067].

[9] P. E. L. Rakow, Stochastic perturbation theory and the gluon condensate PoS LAT2005, 284 (2006), [hep-lat/0510046].

[10] Y. Meurice, The non-perturbative part of the plaquette in quenched QCD, hep-lat/0609005.

[11] A. Denbleyker, D. Du, Y. Meurice (Iowa U.), A. Velytsky, Fisher's zeros of quasi-Gaussian densities of states e-Print: arXiv:0708.0438 [hep-lat]

[12] A. Denbleyker, D. Du, Y. Meurice (Iowa U.), A. Velytsky, Fisher's zeros in $S U$ (2) and $S U(3)$ gluodynamics, in preparation.

[13] A. H. Mueller, Talk given at Workshop on QCD: 20 Years Later, Aachen, Germany, June 1992.

[14] M. Shifman, ITEP Lectures on Particle Physics and Field Theory, World Scientific (2001).

[15] G. Burgio, F. Di Renzo, G. Marchesini, and E. Onofri, $\Lambda^{2}$-contribution to the condensate in lattice gauge theory, Phys. Lett. B422 (1998) 219-226, [hep-ph/9706209].

[16] S. Necco and R. Sommer, The $N(f)=0$ heavy quark potential from short to intermediate distance, Nucl. Phys. B662 (2002) 328, [hep-lat/0108008].

[17] M. Guagnelli, R. Sommer, and H. Wittig, Precision computation of a low-energy reference scale in quenched lattice QCD, Nucl. Phys. B535 (1998) 389, [hep-lat/ 980600 ].

[18] A. Di Giacomo and G. C. Rossi, Extracting the vacuum expectation value of the quantity $<\alpha / \pi G G>$, Phys. Lett. 100B (1981) 481.

[19] M. Falcioni et al., Complex zeroes of the partition function in 4D SU(2), Phys. Lett. B108 (1982) 331.

[20] N. Alves, B. Berg and S. Sanielevici, Spectral density study of the SU(3) deconfining phase transition, Nucl. Phys. B 376 (1992) 218.

[21] A. Denbleyker, D. Du, Y. Meurice, and M. Naides, Definition and parametrization of non-perturbative effects in quenched QCD, PoS LAT2006 (2006) 215 [hep-lat / 0610008 ]. 